\title{
Manejo de los trastornos funcionales digestivos más frecuentes en lactantes sanos
}

\author{
Management of the most frequent functional gastrointestinal \\ disorders in healthy infants
}

Dra. María del C. Toca $a^{a}$ Dra. Patricia Sosa ${ }^{b}$,Dr. Gustavo Apriglianoc, Dra. Raquel Furnes ${ }^{d}$, Dra. Silvia Marchisone ${ }^{e}$, Dra. Mabel Moraf, Dra. Marina Orsi ${ }^{8}$, Dra. Graciela Saiegh, Dr. Omar Tabacco y Dra. Marta Wagener

\section{RESUMEN}

Los lactantes sanos, duranteel primer añodevida, suelen presentar varios síntomas funcionales gastrointestinales, como cólicos, regurgitaciones y constipación. El llanto, tan habitual en esta etapa de la vida, puede interpretarse como dolor o malestar de origen digestivo, y la preocupación por esto genera conductas inapropiadas, como sobrealimentación, interrupción de la lactancia, múltiples cambios de fórmulas, innumerables consultas y medicaciones innecesarias. Elobjetivo de este trabajo es actualizar los conocimientos sobre la fisiopatología de los trastornos funcionales digestivos más frecuentes con el fin de evitar sobrediagnósticos y seleccionar el enfoque terapéutico y las intervenciones nutricionales más apropiadas.

a. Gastroenterología Hepatología.

b. Nutrición Hospital Nacional Profesor Alejandro Posadas.

c. Consultorio Pediátrico Urquiza.

d. Hospital Privado de Córdoba.

e. Gastroenterología y Hepatología del Hospital Infantil de Córdoba.

f. Hospital Materno Infantil de San Isidro.

g. Hospital Italiano de Buenos Aires.

h. Hospital Pediátrico Humberto Notti Mendoza.

i. Sanatorio de Niños de Rosario.

j. Gastroenterología, Hepatología y Nutrición. Hospital de Niños Dr. O. Alassia.

Correspondencia:

Dra. María del Carmen

Toca: mtoca@intramed.net.

Financiamiento:

La escritura de este trabajo fue financiada por Nutricia Bagó.

Conflicto de intereses:

Ninguno que declarar.

Recibido: 18-3-2015

Aceptado: 10-7-2015
Palabrasclave:lactantes, llanto, cólico, regurgitación gástrica, constipación, fórmulas infantiles.

\section{ABSTRACT}

Healthy infants during the first year of life often have multiple functional gastrointestinal symptoms such as colic, regurgitation and constipation. The usual fuzziness and/or crying at this stage of life is interpreted as digestive discomfort or pain, and the corresponding concerns result in unsuitable behaviors as overeating, interruption of breastfeeding, multiple changes of formulas, innumerable queries and unnecessary medications. The aim of this paper is to update knowledge on the pathophysiology of the mostcommon functional gastrointestinal disorders, in order to avoid over diagnosis and select the most appropriate therapeutic approach and convenient nutritional interventions.

Key words: infants, crying, colic, gastric regurgitation, constipation, infant formula.

http:/ /dx.doi.org/10.5546/aap.2015.568

\section{INTRODUCCIÓN}

Los lactantes frecuentemente presentan, durante el primer año de vida, síntomas gastrointestinales por trastornos funcionales, tales como regurgitación, vómitos, constipación, cólicos y llanto. A pesar de que estos niños presentan buen estado clínico, buen desarrollo antropométrico y neuromadurativo, sin anomalías estructurales ni anatómicas ni bioquímicas, se generan frecuentes consultas al pediatra, preocupación en los padres y modificaciones dietarias e indicaciones medicamentosas innecesarias. ${ }^{1}$

Su manejo no puede basarse en la evidencia científica, ya que los trabajos prospectivos, doble ciego, controlados con placebo, son escasos. Por esto, los consensos entre líderes de opinión son el mejor camino para establecer algoritmos de diagnóstico y tratamiento.

Los objetivos de esta actualización son los siguientes: 1) Describir los trastornos funcionales del primer año de vida y su fisiopatología; 2) Describir los algoritmos diagnósticos y terapéuticos dirigidos al pediatra general.

\section{Metodología de trabajo}

Se realizó la búsqueda bibliográfica, se seleccionaron los artículos publicados en los últimos 5 años y se agregaron otros previos de relevancia para el tema. Se establecieron definiciones, bases fisiológicas, diagnóstico y manejo clínico y nutricional.

Se consensuaron los temas analizados. Los coordinadores realizaron el escrito final.

\section{DESARROLLO DEL SISTEMA NERVIOSO ENTÉRICO. PRE- Y POSNATAL} ¿Podemos explicar el origen de los trastornos funcionales del lactante? El sistema nervioso entérico (SNE) 
se establece durante el desarrollo fetal, desde la $4^{\text {ta }}$ a la $7^{\text {ma }}$ semana de embarazo hasta el nacimiento. En períodos finales del embarazo, ya se observa una actividad intestinal coordinada, que incluye diferenciación de subtipos neuronales, formación de proyecciones axonales y el establecimiento de interconexiones funcionales intrínsecas entre nervios, músculo liso y células intersticiales de Cajal, con actividad contráctil refleja intestinal totalmente independiente; de allí que se lo llama "segundo cerebro" o "cerebro pequeño".

En diversas funciones gastrointestinales, el SNE interactúa con el sistema nervioso central (SNC), el sistema nervioso autónomo y la microbiota intestinal, y juntos forman una red de integración que permite señales desde el cerebro al intestino y del intestino al cerebro, llamada “eje cerebro-intestinal". A su vez, el cerebro y el intestino están constantemente comunicados también con la microbiota intestinal, que juega un rol fundamental en esta interacción. ${ }^{2-5}$

Recientes estudios muestran que también durante el período posnatal ocurren modificaciones (maduración, podríamos decir), que permite que el SNE se adapte a situaciones posnatales, como las que requieren el crecimiento del intestino, la adaptación a cambios en la dieta, a situaciones de estrés, a lesión o inflamación intestinal. ${ }^{5}$

\section{LLANTO Y CÓLICOS DEL LACTANTE}

El llanto precede al habla y es la manera en que el recién nacido puede expresar su malestar o su deseo de contacto humano. El lactante responde a la mayoría de sus necesidades con el llanto, respuesta regida por un dominio subcortical del SNC. En los dos primeros meses de edad, el llanto se produce como resultado del inadecuado control del SNC en respuesta a estímulos sensoriales, como variaciones de un proceso normal de maduración y desarrollo del SNC. A medida que aumenta el predominio cortical sobre el subcortical, la respuesta a la demanda es diferente y el llanto disminuye. ${ }^{6}$

El llanto es más frecuente en la última parte del día, tarde-noche. Esta presentación horaria es frecuente y no es casual; se relaciona con el ritmo circadiano y con los niveles de melatonina. ${ }^{7}$

Algunos lactantes presentan llanto inconsolable (ataques imposibles de calmar) entre las 3 semanas y los 5 meses de edad. Este es el disparador más importante de estrés en los padres, que sienten frustración, impotencia, culpabilidad por no poder detenerlo. ${ }^{8}$
Con frecuencia, los padres lo atribuyen a "hambre", con riesgo de sobrealimentar a los niños con el afán de calmarlos y cesación prematura de la lactancia, o a "cólicos", que involucran el tracto gastrointestinal.

Se define "cólico del lactante" al cuadro que se expresa como llanto inconsolable paroxístico, que empieza y termina sin causa aparente, durante 3 horas, 3 días por semana, 3 semanas (regla de 3 de Wessel) o 3 horas por día durante una semana. El cuadro típico comienza en menores de 3 meses $\mathrm{y}$, rara vez, se extiende más allá de los 6 meses. ${ }^{9,10}$

El llanto y el cólico tienen mucho en común. El llanto prolongado produce cólicos -aerofagia- y los cólicos, al producir dolor, causan llanto. Provocan malestar en el niño, pero ambos aseguran la proximidad del cuidador y su supervivencia. ${ }^{9}$

\section{Diagnósticos diferenciales}

Los signos o síntomas de alarma que deben hacer sospechar un origen orgánico son regurgitaciones frecuentes, vómitos y episodios de tos, posición de Sandifer (postura anómala de la cabeza y el cuello -tortícolis- y arqueamiento de la columna vertebral en hiperextensión, que es un recurso para aliviar el dolor que produce el ácido en niños con reflujo gastroesofágico, al facilitar el clearance o vaciamiento esofágico), historia familiar de atopía, síntomas respiratorios o dermatológicos de alergia, sangrado gastrointestinal o signos clínicos de anemia, falla de crecimiento, distensión abdominal, dermatitis perianal y mal estado general. ${ }^{11}$

Las causas orgánicas más importantes que se deben tener en cuenta para descartar en niños con llanto inconsolable son la alergia a las proteínas de leche de vaca (APLV) y, con menor frecuencia, el reflujo gastroesofágico (RGE). ${ }^{12-14}$

En la Tabla 1, se enumeran todas las posibles causas orgánicas de llanto. ${ }^{12}$

\section{ENFOQUE TERAPÉUTICO}

La diversidad de las hipótesis etiológicas del llanto y los cólicos del lactante determinan que no exista un tratamiento etiológico común para todos los pacientes.

\section{Información a los padres}

Es prioritario establecer un vínculo adecuado de comunicación, orientado a la resolución espontánea que se observa a partir $\operatorname{del} 3^{\text {ro }} \mathrm{o} 4^{\text {to }}$ mes de vida del niño. ${ }^{15,16}$

Es de utilidad aconsejar cómo calmar al bebe y cómo mejorar la técnica alimentaria para 
disminuir la aerofagia. ${ }^{17}$ El grado de repercusión familiar, y en su dinámica, permitirá definir qué pacientes requieren intervención interdisciplinaria psicológica o psiquiátrica.

\section{Recomendaciones nutricionales}

La leche materna (LM) constituye la mejor opción en el manejo de niños con cólicos. Nunca debe suspenderse la LM porque el niño tiene cólicos o llanto, ya que contiene oligosacáridos (prebióticos), que favorecen el desarrollo de la microbiota bifidogénica saludable, y factores inmunomoduladores, que favorecen las modificaciones posnatales, lo que permite un adecuado desarrollo madurativo del sistema gastrointestinal. ${ }^{18}$

\section{Fórmulas}

Si bien el rol de la lactosa como generadora de cólicos está cuestionado, a partir de estudios aleatorizados controlados en niños no amamantados, se demostró una mejoría de los síntomas con la utilización de fórmulas con reducción (NO suspensión) de lactosa. Conocido el rol importante como prebiótico que cumple la lactosa, la reducción debe ser mínima y transitoria. ${ }^{11}$
Otras publicaciones utilizan fórmulas fermentadas, con actividad de lactasa, por la presencia de Streptococcus thermophilus o de Bifidobacterium breve (bacterias destruidas por tratamiento térmico posterior), que hidrolizan la lactosa y disminuyen su fermentación colónica. ${ }^{19,20}$

\section{Intervención farmacológica}

Ensayos aleatorizados controlados con placebo no han demostrado que los fármacos simeticona, diciclomina, dicicloverina o cimetropium ofrezcan apropiados márgenes terapéuticos de efectividad y seguridad. Tampoco las medicinas complementarias o alternativas han demostrado beneficios. $^{21-26}$

El RGE no es una etiología frecuente del cólico y llanto del lactante, por lo cual el uso empírico de medicamentos bloqueantes de receptores H2 (ranitidina) o de la producción de ácido (omeprazol) no está indicado, más aún si se considera que producen efectos adversos sobre la microbiota. ${ }^{14}$

Una terapéutica objeto estudio en la actualidad es la utilización de probióticos. El más utilizado en las investigaciones es el Lactobacillus reuteri, especialmente dos cepas, la 55730 y la DSM 17938. Los resultados no son contundentes. ${ }^{27-30}$

TABla 1. Causas orgánicas de llanto

\begin{tabular}{lcc}
\hline Causas orgánicas & Frecuentes & Menos frecuentes \\
\hline Generales & Déficit auditivo o visual & Otitis \\
& Reacción a vacunas & Sepsis \\
& Erupción dentaria prematura & Bacteriemia \\
SNC & Inmadurez del SNC & \\
& (predominio subcortical) & Enfermedades metabólicas \\
& & Meningoencefalitis \\
Sistema digestivo & Hidrocefalia \\
& Reflujo gastroesofágico & Craneoestenosis \\
Sistema respiratorio & Constipación & Malformaciones \\
Sistema cardiovascular & Alergia a la leche de vaca & Síndrome pilórico \\
& Obstrucción de VAS & Bronquiolitis \\
Sistema genitourinario & Catarro de VAS & Neumonía \\
& Arritmias: taquicardia supraventricular & Insuficiencia cardíaca \\
& & Miocarditis \\
& Coartación de aorta
\end{tabular}


Se están desarrollando numerosos trabajos multicéntricos con diversos probióticos, con mayor número de pacientes, que se espera que permitan delinear bien su utilidad.

\section{REGURGITACIÓN}

La regurgitación es el retorno involuntario de secreciones o alimentos ingeridos, dentro o fuera de la boca, mientras que los vómitos son la expulsión violenta del contenido gástrico. En la práctica clínica, se utilizan ambos términos indistintamente. Nos referiremos a regurgitación como manifestación de RGE fisiológico, sin tener en cuenta si es o no expulsado por la boca. ${ }^{31}$

La regurgitación es un trastorno funcional fisiológico que se presenta en niños de 3 semanas a 12 meses de edad, que regurgitan 2 o más veces por día durante, al menos, 3 semanas. Ocurre sin náuseas, hematemesis, aspiración, apneas, falla de crecimiento, dificultad en la alimentación o deglución ni posturas anómalas. ${ }^{10}$

Es más frecuente en el primer mes de vida y disminuye gradualmente a partir del $5 .{ }^{\text {to }}$ mes. Al año de edad, es del $4 \%$ y es infrecuente a los 18 meses. La evolución natural de la regurgitación es más corta en los lactantes amamantados. ${ }^{32,33}$

El niño sano que regurgita se conoce como "regurgitador o vomitador feliz".

En los primeros meses, hay factores que lo predisponen:

- Aerofagia frecuente.

- Baja presión del esfínter esofágico inferior (EEI).

- Capacidad gástrica limitada.

- Incoordinación motora del aparato digestivo en los primeros meses de vida.

- Mala técnica alimentaria. ${ }^{11,31}$

\section{Diagnósticos diferenciales}

Los signos o síntomas de alarma que podrían indicar una causa orgánica son pobre ganancia de peso, deshidratación, anemia, hematemesis, trastornos de deglución, enfermedad respiratoria, rechazo del alimento, irritabilidad/llanto excesivo, diarrea, eczema. ${ }^{31}$

Tienen riesgo aumentado los niños prematuros, niños con retardo madurativo y con anormalidades congénitas orofaríngeas, tórax, pulmones, SNC, corazón y tracto gastrointestinal.

El primer paso diagnóstico es una anamnesis y examen clínico detallados. Si el lactante se alimenta bien, aumenta bien de peso, no tiene irritabilidad ni otras complicaciones, se lo considera RGE no complicado o fisiológico. Los exámenes radiológicos $\mathrm{NO}$ son necesarios. Se aconseja un control periódico para evaluar la probabilidad de nuevos síntomas o signos de alarma. ${ }^{31,34}$

Síntomas de enfermedad por reflujo gastroesofágico: véase Tabla 2.

Causas orgánicas de vómitos: véase Tabla 3.

\section{ENFOQUE TERAPÉUTICO}

1. Información a los padres

Se debe dejar en claro lo siguiente:

Qué es el RGE fisiológico y por qué se resuelve espontáneamente cerca del año de edad.

Cuáles son los signos de alarma ante los que deben consultar a su pediatra.

Fortalecer un buen vínculo madre-hijofamilia. ${ }^{11,35}$

En presencia de síntomas de congestión nasal o dificultad para respirar durante el sueño, como producto de las regurgitaciones frecuentes, deben tomarse medidas generales para mejorarlos. ${ }^{36}$

Medidas conservadoras, como la posición erguida o lateral izquierda en el período posprandial inmediato, pueden mejorar las regurgitaciones. No se recomienda la posición prona por el aumento del riesgo de muerte súbita del lactante. ${ }^{11,34,36}$

Se recomienda NO exponer al lactante al humo del tabaco: la nicotina disminuye la presión del EEI y aumenta las regurgitaciones y el RGE. ${ }^{11,31}$

\section{Recomendaciones nutricionales}

La LM es la mejor alimentación en el manejo de niños con regurgitaciones. Tiene un efecto protector sobre la regurgitación al mejorar el vaciamiento gástrico.

TABLA 2. Signos de sospecha de enfermedad por reflujo gastroesofágico

\author{
Signos de sospecha de ERGE: \\ Pobre ganancia de peso \\ Anemia \\ Hematemesis \\ Trastornos de deglución \\ Rechazo del alimento \\ Irritabilidad/llanto excesivo \\ Respuestas anómalas en la vía aérea \\ Apneas \\ Laringitis \\ Neumonías recurrentes \\ Posturas distónicas
}

ERGE: enfermedad por reflujo gastroesofágico. 
Nunca debe suspenderse la LM con el objeto de espesar la leche. ${ }^{37}$

\section{Fórmulas}

En lactantes NO amamantados, el espesamiento de la fórmula tiene efectos sobre el síntoma al disminuir la frecuencia y la cantidad de regurgitaciones. Estudios han demostrado que reduce el número de episodios de reflujo NO ácidos ( $\mathrm{pH}>4)$, y disminuye la altura que alcanza el reflujo en el esófago, y que no es efectivo en pacientes con enfermedad por RGE, estudiados con pHmetría e impedanciometría. ${ }^{38-43}$

Se utilizan como espesantes harina de semilla de algarrobo, almidón de maíz, papa o arroz.

La harina de semilla de algarrobo no es hidrolizada por amilasas salivares y permanece con poder espesante en el estómago, no tiene valor nutricional, no aumenta las calorías y tiene efecto de fibra, lo que evita la constipación de otros espesantes. ${ }^{38,43,44}$

No se recomienda el uso de las fórmulas espesadas en forma casera, ya que las modificaciones en la osmolaridad y la concentración del alimento, pueden empeorar el vaciamiento gástrico. ${ }^{11,45}$

En cuanto al volumen, la ingesta frecuente y el exceso de volumen (mayor de $20 \mathrm{ml} / \mathrm{kg}$ de peso) pueden aumentar el número de regurgitaciones. ${ }^{41}$

A partir de los 6 meses de edad, es recomendable que la alimentación complementaria tenga menor contenido de grasas para disminuir la posibilidad de RGE al acelerar el vaciamiento gástrico y disminuir la secreción de ácido. ${ }^{34,35,41}$

\section{CONSTIPACIÓN. DISQUECIA Comportamiento evacuatorio normal en niños menores de 2 años}

- Los lactantes alimentados con LM tienen un promedio de 4-6 deposiciones por día, de consistencia líquida, de color verde y/o amarillo, nunca blancas ni rojas ni negras.

- A lo largo del $1^{\mathrm{er}}$ año, el número, la consistencia y el color se van modificando para ser de 1 a 2 veces por día, consistentes y marrones con la alimentación complementaria.

- Los niños alimentados con LM pueden pasar varios días sin evacuar y no implica enfermedad si luego hacen heces blandas, sin dolor y de aspecto habitual al de los bebes amamantados. ${ }^{46}$

\section{Constipación}

Dificultad en la evacuación o vaciamiento incompleto del colon y el recto, expresado como la eliminación de heces duras e infrecuentes, cuya causa, en el 95\% de los casos, es funcional o idiopática. Casi el 40\% comienza al año de vida.

\section{Disquecia del lactante}

Es un trastorno evacuatorio funcional, transitorio y benigno de los lactantes menores de 6 meses, sanos, alimentados con LM, que

Tabla 3. Causas orgánicas de vómitos

\begin{tabular}{cl}
\hline Causas orgánicas & Síntomas \\
\hline Enfermedad gastrointestinal & Vómitos biliosos \\
APLV. Esofagitis eosinofílica & Sangrado gastrointestinal \\
Obstrucción gastrointestinal & Vómitos en chorro \\
Anomalías anatómicas & Vómitos que comienzan luego de los 6 meses \\
Hipertrofia pilórica & Constipación \\
Malrotación intestinal & Diarrea \\
Membrana duodenal & Distensión abdominal \\
& Hepatoesplenomegalia \\
& Fontanela hipertensa \\
Enfermedad neurológica o sistémica & Macro/microcefalia \\
& Convulsiones \\
& Sospecha de síndrome metabólico o genético \\
& Infecciones crónicas \\
\hline Síntomas no específicos & Retraso del crecimiento \\
& Fiebre \\
& Letargo \\
\hline
\end{tabular}

APLV: alergia a las proteínas de leche de vaca. Sospecha de causas orgánicas. Signos de alarma ante vómitos. 
presentan retardo evacuatorio (por 3 o más días), con maniobras de empuje, enrojecimiento facial, sin evacuación durante unos minutos, con llanto intenso que cesa con la expulsión de heces blandas o semilíquidas.

Está causado por la falla en la coordinación entre el aumento de la presión intraabdominal y la relajación del piso pélvico. Las contracciones de músculos abdominales y glúteos no son efectivas para lograr la salida de la materia fecal.

No requiere ninguna medida terapéutica y se resuelve espontáneamente en unas semanas, una vez que el lactante logra una efectiva dinámica defecatoria.

Genera mucha preocupación en los padres, que intentan mejorarla con maniobras de estimulación anal (supositorios, cremas y/o sondas), que deben desaconsejarse. ${ }^{1}$

\section{Estreñimiento funcional crónico}

Refiere a la presencia de deposiciones duras de gran tamaño, evacuaciones menos de 2 a 3 veces por semana, con actitud de retención por miedo al dolor de, al menos, 1 mes de evolución. ${ }^{10}$

\section{Etiología de constipación}

\section{- Dietéticos}

Destete. Las deposiciones duras se presentan en solo el $1,1 \%$ en niños amamantados versus el 9,2\% en niños alimentados con fórmulas. Las fórmulas con contenido de ácidos grasos (AG), en posición alfa, son responsables del aumento de consistencia de las heces porque la lipasa pancreática no puede hidrolizar estos AG, por lo que no pueden ser absorbidos y son eliminados en forma de jabones cálcicos insolubles, que endurecen las heces. En cambio, en la LM, el 70\% de los AG se localizan en posición beta. ${ }^{47}$

Otras causas: inadecuada preparación de fórmulas o poca ingesta de agua. Consumo alto de cereales y lácteos. Baja ingesta de vegetales.

- Dolor por lesiones anales o perianales.

- Predisposición familiar: presente en el 50\% de los lactantes con estreñimiento antes de los 6 meses.

- Medicaciones: antitusígenos, derivados opioides, atropina. ${ }^{11,48}$

\section{Diagnósticos diferenciales}

Se debe iniciar el estudio con una minuciosa anamnesis y examen físico.

Exámenes complementarios: no son necesarios.
Síntomas y signos de alarma:

- Retraso en la evacuación de meconio o evacuación inducida. El 99\% elimina meconio en las primeras $48 \mathrm{~h}$ de vida y también el $50 \%$ de los pacientes con enfermedad de Hirschsprung, por lo que no excluye el diagnóstico.

- Comienzo precoz en período neonatal.

- Malformaciones anales, atonía del esfínter, alteraciones de la columna lumbosacra, fosita pilonidal o mechón de pelo, desviación de la línea glútea, nalgas planas.

- Falla de crecimiento, vómitos, distensión abdominal, fiebre, trastornos miccionales.

- Alteraciones del desarrollo madurativo, hipotonía o espasticidad.

- Falta de respuesta terapéutica. ${ }^{11,48}$

\section{ENFOQUE TERAPEÚTICO}

1. Información a los padres Se debe disminuir la ansiedad familiar informando sobre la posibilidad de resolución espontánea al año de edad y alertar sobre los signos de alarma. ${ }^{11}$

2. Intervenciones nutricionales

La LM es la mejor alimentación en el manejo de niños con constipación. Debe asegurarse que el aporte sea el adecuado, que se corrobora con buen aumento de peso, ya que el hipoaporte alimentario puede manifestarse con reducción del número de deposiciones.

En lactantes menores de 6 meses alimentados con fórmula, se debe ofrecer agua entre raciones $(5-10 \mathrm{ml} / \mathrm{kg} /$ día). Se recomienda el uso de fórmulas con oligosacáridos (prebióticos) y con AG (palmitato) en posición beta, semejantes a los de la LM. ${ }^{47-51}$

En lactantes mayores de 6 meses, se debe asegurar la ingesta de fibra -los jugos de fruta (pera, manzana y ciruela) contienen sorbitol con poder laxante-, vegetales, cereales integrales y legumbres.

Se debe ajustar la ingesta de lácteos y derivados, ya que su exceso puede favorecer la constipación.

3. Tratamiento medicamentoso

Laxantes osmóticos: polietilenglicol o lactulosa.

La dosis de laxantes debe ajustarse hasta conseguir 1-2 deposiciones blandas al día sin esfuerzo. $^{48}$ 


\section{CONCLUSIONES}

La presentación de síntomas gastrointestinales leves y de etiología funcional es frecuente en los lactantes. Su diagnóstico se basa en una adecuada anamnesis y un minucioso examen físico. Es excepcional la necesidad de exámenes complementarios. La mejor recomendación nutricional es la leche materna. El éxito del manejo se basa en informar al núcleo familiar de su evolución benigna y favorable.

\section{REFERENCIAS}

1. Di Lorenzo C. Other functional gastrointestinal disorders in infants and young children. J Pediatr Gastroenterol Nutr 2013;57(Suppl 1):S36-8.

2. Burns A, Thapar N. Development and postnatal changes in the Enteric Nervous System. J Pediatr Gastroenterol Nutr 2013;57(Suppl 1):S4-8.

3. Soret R, Chevalier J, De Coppet P, Poupeau G, et al. Short chain fatty acids regulate the enteric neurons and control gastrointestinal motility in rats. Gastroenterology 2010;138(5):1772-82.

4. Indrio F, Riezzo G, Raimondi F, Di Mauro A, Francavilla R. Microbiota involvement in the gut-brain axis. J Pediatr Gastroenterol Nutr 2013;57(Suppl 1):S11-5.

5. Di Lorenzo C. Impact of early life events on pediatric functional gastrointestinal disorders. J Pediatr Gastroenterol Nutr 2013;57(Suppl 1):S15-8.

6. James-Roberts I, Di Lorenzo C. Background and scope. J Pediatr Gastroenterol Nutr 2013;57(Suppl 1):S2-3.

7. Leuchter R, Darque A, Hüppi P. Brain maduration, early sensory processing, and infant colic. J Pediatr Gastroenterol Nutr 2013;57(Suppl 1):S18-25.

8. James-Roberts I, Álvarez M, Hovish K. Emergence of a developmental explanation for prolonged crying in 1- to 4- month old infants: Review of the evidence. J Pediatr Gastroenterol Nutr 2013;57(Suppl 1):S30-6.

9. Shamir R, James Roberts I, Di Lorenzo C, Burns A, Thapar N, et al. Infant crying, colic, and gastrointestinal discomfort in early childhood: a review of the evidence and most plausible mechanisms. J Pediatr Gastroenterol Nutr 2013;57:S1.

10. Hyman PE, Milla PJ, Benninga MA, Davidson GP, et al. Childhood functional gastrointestinal disorders: neonate/ toddler. Gastroenterology 2006;130(5):1519-26.

11. Vandenplas Y, Gutiérrez-Castrellón P, Velasco-Benítez C, Palacios J, et al. Practical algorithms for managing common gastrointestinal symptoms in infants. Nutrition 2013;29(1):184-94.

12. Freedman SB, Al-Harthy N, Thull-Freedman J. The crying infant: diagnostic testing and frecuency of serious underlying disease. Pediatrics 2009;123(3):841-8.

13. Heine R. Cow's-Milk allergy and lactose. Malabsorption in infants with colic. J Pediatr Gastroenterol Nutr 2013;57(Suppl 1):S25-7.

14. Indrio F, Riezzo G, Raimondi F, Di Mauro A, Francavilla R. Gut motility alterations in neonates and young infants: Relation to colic? J Pediatr Gastroenterol Nutr 2013;57(Suppl 1):S9-11.

15. Lucassen PL, Assendelft WJ, Gubbels JW, Van Eijk JT, et al. Effectiveness of treatments for infantile colic: systematic review. BMJ 1998;316(7144):1563-9.

16. Taubman B. Parental counseling compared with elimination of cow's milk or soy milk protein for the treatment of infant colic syndrome: a randomized trial. Pediatrics 1988;81(6):756-61.

17. Kheir AE. Infantile colic, facts and fiction. Ital J Pediatr 2012;38:34.

18. Bellaïche M, Levy M, Jung C. Treatments for Infant Colic. J Pediatr Gastroenterol Nutr 2013;57(Suppl 1):S27-30.

19. Agostoni C, Goulet O, Kolacek S, Koletzko B, et al. Fermented infant formulae without live bacteria. J Ped Gastroenterol Nutr 2007;44(3):392-7.

20. Roy P, Aubert-Jacquin C, Avart C, Gontier C. Benefits of a thickened infant formula with lactase activity in the management of bening digestive disorders in newborns. Arch Pediatr 2004;11(12):1546-54.

21. Danielsson B, Hwang CP. Treatment of infantile colic with surface active substance (simethicone). Acta Paediatr Scand 1985;74(3):446-50.

22. Metcalf TJ, Irons TG, Sher LD, Young PC. Simethicone in the treatment of infant colic: a randomized, placebocontrolled, multicenter trial. Pediatrics 1994;94(1):29-34.

23. Weizman Z, Alkrinawi S, Goldfarb D, Bitran C. Efficacy of herbal tea preparation in infantile colic. J Pediatr 1993;122(4):650-2.

24. Alexandrovich I, Rakovitskaya O, Kolmo E, Sidorova T, Shushunov S. The effect of fennel (Foeniculum vulgare) seedoil emulsion in infantile colic: a randomized, placebocontrolled study. Altern Ther Health Med 2003;9(4):58-61.

25. Savino F, Cresi F, Castagno E, Silvestro L, Oggero R. A randomized double-blind placebo-controlled trial of a standardized extract of Matricariae recutita, Foeniculum vulgare and Melissa officinalis (ColiMil) in the treatment of breastfed colicky infants. Phytother Res 2005;19(4):335-40.

26. Ize-Ludlow D, Ragone S, Bruck IS, Duchowny M, Peña $\mathrm{BM}$. Neurotoxicities in infants seen with the consumption of star anise tea. Pediatrics 2004;114(5):e653-6.

27. Anabrees J, Indrio F, Paes B, AlFaleh K. Probiotics for infantile colic: a systematic review. BMC Pediatr 2013;13:186.

28. Sung V,Hiscock H, Tang M, Mensah F, etal. Treating infant colic with the probiotic Lactobacillus reuteri: double blind, placebo controlled randomised trial. BMJ 2014;348:g2107.

29. Savino F, Cordisco L, Tarasco V, Palumeri E, et al. Lactobacillus reuteri DSM 17938 In infantile colic: a randomized, double-blind, placebo-controlled trial. Pediatrics 2010;126(3):e526-33.

30. Szajewska H, Gyrczuk E, Horvath A. Lactobacillus reuteri DSM 17938 For the management of infantile colic in breastfedinfants: a randomized, double-blind, placebocontrolled trial. J Pediatr 2013;162(2):257-62.

31. Vandenplas Y, Roudolh CD, Di Lorenzo C, Hassall E, et al. Pediatric Gastroesophageal Reflux Clinical Practice Guidelines: Joint Recommendations of the North American Society for Pediatric Gastroenterology, Hepatology, and Nutrition (NASPGHAN) and the European Society for Pediatric Gastroenterology, Hepatology, and Nutrition (ESPGHAN). J Pediatr Gastroenterol Nutr 2009;49(4):498-547.

32. Campanozzi A, Boccia G, Pensabene L, Panetta F, et al. Prevalence and natural history of gastroesophageal reflux: pediatric prospective survey. Pediatrics 2009;123(3):779-83.

33. Hegar B, Dewanti N, Kadim L, Alatas S, et al. Natural evolution of regurgitation in healthy infants. Acta Pædiatr 2009;98(7):1189-93.

34. Lightdale J, Gremse D. Gastroesophageal reflux: Management Guidance for the Pediatrician. Pediatrics 2013;131(5):e1684-95.

35. Orsi M. Reflujo gastroesofágico en Pediatría. En: Sociedad Argentina de Pediatría. Pronap 2011;(4):15-33.

36. Orenstein SR, McGowan JD. Efficacy of conservative therapy as taught in the primary care setting for symptoms 
suggesting infant gastroesophageal reflux. J Pediatr 2008;152(3):310-4.

37. Heackock HJ, Jeffery HE, Baker JL, Page M. Influence of breast versus formula milk on physiological GER in healthy, newborn infants. J Pediatr Gastroenterol Nutr 1992; 14(1):41-6.

38. Aggett PJ,Agostoni C, Goulet O,HernellO, etal. Antireflux or antiregurgitation milk products for infants and young children: a commentary by the ESPGHAN Committee on Nutrition. J Pediatr Gastroenterol Nutr 2002;34(5):496-8.

39. Orenstein SR, Magill HL, Brooks P. Thickening of infants feeding for therapy of gastroesophageal reflux. J Pediatr 1987;110(2):181-6.

40. Craig WR, Hanlon-Dearman A, SinclairC, TabackS, Moffatt M. Metoclopramide, thickened feedings, and positioning for GER in children under two years. Cochrane Database Syst Rev 2004;(4):CD003502.

41. Khoshoo V, Ross G, Brown S, Edell D. Smaller volume, thickened formulas in the management of gastroesophageal reflux in thriving infants. J Pediatr Gastroenterol Nutr 2000;31(5):554-6.

42. Martin AJ, Pratt N, Kennedy JD, Ryan P, et al. Natural history and familial relationships of infant spilling to 9 years of age. Pediatrics 2002;109(6):1061-7.

43. Wenzl TG, Schneider S, Scheele F, Silny J, et al. Effects of thickened feeding on gastroesophageal reflux in infants: a placebo-controlled crossover study using intraluminal Impedance. Pediatrics 2003;111(4 Pt 1): e355-9.

44. Borelli O, Salvia G, Campanozzi A, Franco M T, et al. Use of a new thickened formula for treatment of symptomatic gastroesophageal reflux in infants. Ital J Gastroenterol Hepatol 1997;29(3):237-42.

45. Miyazawa R, Tomomasa T, Kaneko H, Morikawa A. Effect of formula thickened with locust bean gum on gastric emptying in infants. J Paediatr Child Health 2006;42(12):80812.

46. Tunc V, Camurdan A, lhan M, Sahin F, Bevazora U. Factors associated with defecation patterns in 0-24-month-old children. Eur J Pediatr 2008;167(12):1357-62.

47. Ferrer Lorente B, Miñana V, Dalmau Serra J. Indicaciones para las fórmulas lácteas especiales: fórmulas para problemas «menores», fórmulas sin lactosa y fórmulas de proteína de soja. Acta Pediatr Esp 2009;67(7):333-7.

48. Tabbers MM, Di Lorenzo C, Berger MY, Faure C, et al. Evaluation and treatment of functional constipation in infants and children: evidence-based recommendations from ESPGHAN and NASPGHAN. J Pediatr Gastroenterol Nutr 2014:58(2):258-74.

49. Euler A, Mitchell D, Kline R, Pickering L. Prebiotic effect of fructo-oligosaccharide supplemented term infant formula at two concentrations compared with unsupplemented formula and human milk. J Pediatr Gastroenterol Nutr 2005;40(2):157-64.

50. Chirdo F, Menéndez A, Portela M, Sosa P, et al. Prebióticos en salud infantil. Arch Argent Pediatr 2011;109(1):49-54.

51. Ziegler E, Vanderhoof J, Petschow B, Mitmesser S, et al. Term infants fed formula supplemented with selected blends of prebiotics grow normally and have soft stools similar to those reported for breast-fed infants. J Pediatr Gastroenterol Nutr 2007;44(3):359-64. 